\title{
Transportation into Narrative Worlds
}

\author{
Melanie C. Green
}

From centuries-old fables to modern television series, entertainmenteducation often involves telling stories. And stories work best when audiences are immersed in them: readers or viewers leave the real world behind and step into the world created by the authors. When people are transported to these narrative worlds and their interest is captivated, they may experience strong emotions and have vivid mental images. They may not notice their immediate surroundings or the passage of time. This feeling of being "lost in a story" is the core of narrative transportation theory.

Narrative transportation has been defined as a combination of attention, imagery, and feelings, in which an individual becomes immersed in a narrative world (Green \& Brock, 2000; Green \& Brock, 2002). Individuals who are more transported are more likely to adopt beliefs, attitudes, and behaviors that are implied by the story (e.g., Murphy et al., 2013). Even though stories typically show specific people and events, audiences tend to generalize the lessons from the stories and apply them to real-life situations. These effects have been demonstrated across a variety of different topics and issues, including health, consumer products, and social issues. A recent meta-analysis has summarized these effects (Van Laer, de Ruyter,

M. C. Green $(\bowtie)$

University at Buffalo, The State University of New York, Buffalo, NY, USA e-mail: mcgreen2@buffalo.edu

(C) The Author(s) 2021

L. B. Frank, P. Falzone (eds.), Entertainment-Education Behind the

Scenes, https://doi.org/10.1007/978-3-030-63614-2_6 
Visconti, \& Wetzels, 2014; see also Braddock \& Dillard, 2016 for a metaanalysis of narrative effects more broadly).

Transportation can also occur across different media. Individuals can be transported into books, movies, spoken stories, or even virtual reality. Research thus far does not indicate a consistent advantage for any particular medium (Braddock \& Dillard, 2016; De Graaf, Hoeken, Sanders, \& Beentjes, 2012). These results may imply that the content of the story itself is the most important element, so entertainment-education professionals can choose the medium that is most appropriate or practical for their audience or that they believe will convey their story in the most effective way. For example, production costs are often much lower for print narratives than video narratives, and some forms of media are easier to distribute than others, especially if audience members may not have particular devices (e.g., they do not have computers or televisions). However, it is also possible that even if the outcomes across media are similar, the psychological processes evoked by different media may be somewhat different. Walter, Murphy, Frank, and Baezconde-Garbanati (2017) found that a video version of a narrative created higher levels of cognitive and emotional involvement than a print version, but also led to higher reactance. Individual differences among audience members may also make one format more or less effective than another. For instance, people who enjoy exerting cognitive effort (those who are high in need for cognition) may be more transported into texts versus films, whereas those who prefer to put in less effort may be more transported into video narratives (Green et al., 2008).

Similarly, transportation can happen whether a story is about actual events (such as a documentary) or whether it is the creation of an author's imagination. Transportation and persuasion can occur in both factual and fictional narratives. For fictional narratives, psychological plausibility appears to be important - even if the characters do not actually exist, audiences should believe that they could exist or that these situations could occur. Fictional narratives do not have to be strictly realistic, however; a story could take place in outer space or a fantasy world, as long as the events develop in a way that is consistent with the rules established within that narrative world and the characters act the way real people would act. For example, fantasy stories such as Harry Potter have shown promise in reducing prejudice (Vezzali, Stathi, Giovannini, Capozza, \& Trifiletti, 2015). 
Furthermore, although longer stories may provide more opportunity for audiences to become deeply transported, research suggests that even relatively brief and simple narratives, such as one-page advertisements or short stories, can be transporting (e.g., Escalas, 2004; Mazzocco, Green, Sasota, \& Jones, 2010). These shorter stories may benefit from drawing on more universal themes or well-known story structures (e.g., success at overcoming an obstacle; the power of love). In practice, the length of an entertainment-education intervention may be determined in part by the context in which the intervention will be delivered. For example, our team developed a heart attack awareness intervention game that was originally intended to be delivered in waiting rooms at doctors' offices; this constraint meant that it needed to be quite short to ensure that individuals could complete it. When we changed our delivery platform to the Internet, we were able to expand the length of the game. Similarly, in online research studies, our participants do not generally want to read lengthy narratives; however, there are many examples of successful web, television, and radio series that capture audience attention for hours or even across years.

Narrative transportation shares some similarities with other types of immersive experiences. For example, identification and transportation are both mechanisms through which a narrative can change reader's attitudes and beliefs. However, identification occurs when readers are able to experience the narrative through the perspective of a story character (Busselle \& Bilandzic, 2009; Cohen, 2001), whereas transportation is a more general immersion in the story world. Identification can be conceptualized as the reader adopting the goals and motivations of the character. Identification and transportation are often, but not always, highly correlated. Indeed, a challenge for entertainment-education is that in narratives with multiple characters, some audience members may identify with characters that are "bad" role models or who engage in negative behaviors.

\section{How Does Transportation Lead to Change?}

There are several ways that transportation may lead to attitude and behavior change. These mechanisms include reduced counterarguing, connections with characters, increases in perceived realism, mental imagery of story events, and emotional engagement. 


\section{Reduced Counterarguing}

One barrier to attitude or behavior change is that individuals may resist or argue against a message. Because they may seem less threatening or more accessible than traditional educational messages, stories or entertainmenteducation can help overcome this resistance. Transporting stories seem to create a more open, accepting mindset for individuals.

In some cases, transported individuals may not be aware that a narrative is persuasive. Due to the entertaining nature of some narratives, readers do not expect to be influenced and thus spend less time actively considering the embedded message of the story. Attitudes and behaviors depicted in the narrative may thus be accepted by the reader without much attention. Furthermore, transportation may reduce the motivation to counterargue or argue against the message or implications of a story. When readers are transported in an entertaining narrative, they may not want to interrupt their enjoyment to argue with the story (i.e., the story's events, claims, or overall message). Counterarguing can therefore be reduced even in cases where the persuasive intent is more obvious (e.g., the story of a cancer survivor urging others to quit smoking).

However, depending on the type of story, sometimes counterarguing can be a sign of engagement: individuals may argue against the claims or actions of villains or negative role models, thus supporting the main message or moral of the narrative.

\section{Connections with Characters}

Individuals may see themselves in story characters, may come to see story characters as friends, or may admire the characters. Through these types of connections, audience members may also change their attitudes and beliefs in accordance with those of a narrative character, or story protagonist. Audience members see and relate to the experiences of the characters, and those experiences may influence how the audience thinks or acts.

Identification with, and liking of, story characters has been shown to increase the adoption of beliefs advocated by the character (De Graaf et al., 2012). Readers may even alter their self-concept to become more similar to characters they liked or identified with, at least temporarily. Although transportation is distinct from identification, transportation is often correlated with identification and liking for protagonists. Additionally, even negative characters (villains) may also lead to attitude change by 
inspiring readers or viewers to reject the views or actions of such characters. For example, in our initial development of a story to increase children's physical activity through active video games $(\mathrm{Lu}$, Green, \& Thompson, 2019), most participants said they would be inspired to be active by running, jumping, and playing like the story's heroes (twins with superpowers), but a small number said they would be motivated for physical activity by the villain - to be able to defeat him!

One related way in which a narrative might prompt self-examination and change is by reminding readers of experiences in their own lives that relate to those in the narrative. Strange and Leung (1999) highlighted the role of "remindings" - links between story content and the reader's past personal or media-based experiences-in narrative impact. Individuals who were more immersed in a story showed greater generalization of the beliefs implied by the story, but additionally, whether or not the story brought to mind events from the readers' real lives seemed to be important in determining narrative impact.

\section{Mental Imagery}

The transportation-imagery model (Green \& Brock, 2002) highlights the role of visual imagery in transportation-based belief change. The experience of being transported into a story links the vivid images with beliefs implied by the story (e.g., the image of a person with a smoking-related illness may be linked with beliefs about the importance of quitting smoking). This connection between images and beliefs may be one basis for the power of narrative persuasion. Additionally, over time, the memory or use of the image may remind people of the story as a whole, thus reinforcing the story-related beliefs. Thus, narratives that inspire the formation of rich mental images can increase the persuasive power of a story. This imagery can be created by descriptions in a text or presented visually. For example, in one of our studies using manga comics to encourage more fruit consumption, the illustrations showed the main character appearing noticeably stronger and more energetic after eating fruit (Leung, Green, Tate, Cai, \& Ammerman, 2017).

\section{Emotional Engagement}

The core of many stories is the feelings they inspire: stories can make us cheer, laugh, or cry. Narratives are particularly powerful when they evoke 
strong emotions. Current research is exploring not only the actual emotions evoked by a narrative, but also the pattern of emotional experience that may occur as readers progress through a story (emotional flow; Nabi and Green, 2015). A change from one emotional tone to another across the course of a story can help maintain interest for audiences, particularly for longer narratives. For example, stories can create and then resolve suspense about the outcomes. However, recent research suggests that emotional shifts may be less effective in some kinds of shorter narratives, perhaps particularly when the emotions are expressed by the character or narrator rather than implied by the events in the story (Ophir, Sangalang, \& Cappella, 2021).

\section{Story Structure/Causality}

Research by Dahlstrom (2012) suggests that events that are part of the cause-and-effect structure of the story are better recalled and more persuasive, even over time, than less-central story elements. Thus, it may be most effective to have the intended persuasive or educational message as part of the main plot of a story, rather than as a digression or something merely mentioned by the characters.

However, one possible consideration is that stories that appear too manipulative may inspire reactance; audiences may feel as though they are being pushed too hard to accept a particular position, and therefore they may reject the message. In some cases, then, a subtler approach such as including the persuasive message as a less central part of the story may have benefits. Indeed, early theorizing promoted narratives as a way to disguise or minimize persuasive intent. However, although stories can reduce resistance to persuasion in this way, there are many examples of entertainment-education that are effective even though the persuasive intent is obvious, particularly if the message is one that the audiences would generally support (e.g., disease prevention).

\section{How Is Transportation Measured and Manipulated?}

In research contexts, transportation is typically measured with a self-report scale. After reading a story, individuals are given a set of statements such as, "The narrative affected me emotionally." The scale includes items about emotional involvement in the story, cognitive attention to the story, feelings of suspense, lack of awareness of surroundings, and mental 
imagery. The original scale included 15 items, including 11 general transportation items and 4 imagery items all relating to a specific narrative, measured on a seven-point scale ranging from "not at all" to "very much."

The 15-item transportation scale was refined to a short form scale with six items (Appel, Gnambs, Richter, \& Green, 2015). The short form may be especially useful for field settings or other contexts where questionnaire brevity is important. The six-item Transportation Scale-Short Form (TS$\mathrm{SF}$ ) is as sensitive as the long form and is available in English and German.

A closely related measure is Busselle and Bilandzic's (2009) narrative engagement scale, which measures four dimensions of engagement in narratives: narrative understanding, attentional focus, emotional engagement, and narrative presence. This scale tends to be highly correlated with measures of transportation, but it is useful in cases where researchers want to focus on specific dimensions of the narrative experience.

Entertainment-education practitioners may not usually have the opportunity to manipulate transportation, but studies suggest that transportation can also be manipulated through pre-narrative instructions (e.g., to relax and enjoy the narrative) or information (e.g., a positive vs. a negative review of the story). One implication is that creating positive expectations for the story may be helpful. However, narrative quality seems to be the strongest determinant of transportation (see Tukachinsky, 2014, for a review).

\section{What Determines Whether Individuals Will Be Transported?}

\section{Features of the Story}

Some stories are better than others. In general, a high-quality story will be more transporting than a low-quality story. Kreuter et al. (2007) suggested a list of features that might contribute to high-quality narratives. One feature is the coherence of the story, where a story provides clear and sensible links between story events, character actions, and other elements of the narrative. Others included character development, the emotional intensity or range of the story, suspense or dramatic tension, and psychological realism (e.g., the plot and characters should be plausible even if the story takes place within a fantasy world). According to Kreuter et al. (2007), features of high-quality narratives could also include cultural 
appropriateness (use of conventions and language familiar to the intended audience) and strong production values (particularly for visual narratives such as movies or television programs).

Busselle and Bilandzic's theory of narrative engagement (a concept very similar to transportation) suggests that immersion or engagement in a narrative arises from the process of mentally simulating or imagining the narrative events (see also Mar and Oatley, 2008). If the flow of this simulation is interrupted by an error or a lack of realism in the text, then the feeling of immersion is lost (and indeed, individuals may become skeptical of the story's claims). The implication for message design is that stories should avoid elements that would be inconsistent with users' knowledge of either the real world or the established rules of a fictional or narrative world.

\section{Match Between the Audience and the Story}

The more familiar a reader is with the material in a narrative, the easier it is for him or her to be transported in it. For example, in one study, readers who were members of fraternities or sororities (social clubs at universities in the United States) were more transported into a story that was set at a fraternity (Green, 2004). There is also some evidence that greater feelings of mental fluency or ease of processing may lead to greater transportation (Vaughn, Childs, Maschinski, Nino, and Ellsworth, 2010). For example, individuals who read a story set in winter during the winter were more transported into it than people who read the same story in the springtime. The idea is that concepts related to winter were more accessible in people's minds in the wintertime, thus making the story easier to imagine. Therefore, creating points of similarity between the audience and the story can be helpful in creating transportation.

Reader preferences can also affect responses. Individuals often have preferences for different genres and types of stories: some individuals may love romance stories whereas others prefer science fiction. People are more likely to enjoy and become transported into their preferred genres.

\section{Features of the Audience}

Some individuals are more likely to become immersed in stories than others, an individual difference that has been called transportability. Highly transportable individuals can become easily immersed in stories, even brief 
ones. Additionally, an individual difference called need for affect (how much people want to experience strong emotions) may also affect story immersion. People who are high in need for affect tend to enjoy and seek out emotion-evoking experiences. They enjoy feeling extremes of happiness, sadness, and other emotions. Because stories tend to show emotioninducing events, such people are also more likely to become transported into (and persuaded by) stories.

Need for cognition is an individual difference in how much people enjoy exerting cognitive effort (Cacioppo, Petty, and Kao, 1984). In other words, people who are high in need for cognition like to think about things, even issues that do not directly affect them. For instance, they might like solving puzzles or debating social issues. People high in need for cognition are more likely to enjoy media that requires more mental effort (e.g., reading a book compared to watching a television show). Such individuals also may be more likely to enjoy interactive narratives, which allow (or require) the reader to make choices to determine which way the story will go (Green \& Jenkins, 2014). Interactive narratives require a higher degree of thinking or engagement.

Thus, although stories and games can be a very effective method of communication, even the best stories may not work for everyone.

\section{ETHical IMPLications of Using STORIES}

Stories can be a powerful and beneficial tool for change. However, like any form of persuasion, stories can promote negative messages as well as positive ones. Creators should be careful about the unintended messages their stories might send. For example, people are very sensitive to descriptive norms - what they believe other people are doing. Therefore, in their efforts to discourage particular behaviors, entertainment-education programs should not make it appear as if most people are engaging in those undesired behaviors (e.g., "everyone" is having unsafe sex and texting while driving).

Additionally, particularly in health contexts, care should be taken so that individuals are not unduly influenced by a story that may not be as relevant to their own situation. In our work, for example, individuals making a hypothetical treatment decision were influenced not only by relevant story content (the match of the patient's risk factors with a story they read) but also by irrelevant details (whether the person in the story had similar hobbies to the target patient; Simons \& Green, 2013). More 
broadly, audience members may have difficulty separating out which parts of the story should be generalized and which should not, or may be distracted by elements of the story which are not relevant to the intended message.

Finally, there may be some contexts where stories are less appropriate; individuals may need or prefer straightforward information rather than an entertainment-education approach. In some cases, a story may be viewed as less credible or may not be able to present all of the necessary facts.

\section{Lessons Learned and Best Practices}

Every theory has boundary conditions. Interventions have the best chance of succeeding when they are guided by theory. Theory can help provide an understanding of the psychological processes that audience members experience. It can help message designers focus on important elements of their messages. However, every theory has boundary conditions-circumstances in which they may not work. So, some theories may be more useful than others for particular topics or situations. For example, our informal observations have been that transportation does not seem to be the main influence on persuasion for stories where the persuasive message is conveyed simply by the statements of the characters, rather than implied by the story events. Transportation works better when stories "show, not tell."

Similarly, although stories can be very powerful, there are some cases where simple informational messages may be preferred. Stories may be misunderstood by the audience, or the main educational point may be overlooked due to audience focus on other aspects of the story.

Many paths to transportation. There are a variety of features that can make stories transporting. A story might have an exciting, suspenseful plot. It might provide deep insights into characters. Or it might have beautiful animation or impressive special effects. Stories do not necessarily have to have each of these elements-stories can be transporting in different ways. For example, the show South Park was very popular despite not having a sophisticated animation style. There are different pathways to reach the same goal of immersing the audience.

Similarly, there are multiple paths to allow audiences to connect with characters. In one game intervention that our team developed, we created a variety of characters to match our target audience on race and gender (e.g., one character was a white woman, one was a Hispanic man, and one 
was an African-American man). Of course, representation is important, especially when one is trying to reach an under-represented target audience. However, it turned out that one character was the clear favorite among the majority of our players: an older, African-American character named Big Joe. Big Joe ran a pizza shop, and his dialogue showed that he had a strong sense of responsibility and deeply cared for his customers. The voice actor who played the role also did a fantastic job of conveying a sense of kindness and warmth. Many of our players noted that Big Joe reminded them of their fathers or grandfathers. The similarity in values and priorities outweighed the similarities or differences in demographic variables such as age, gender, or race.

Narrative quality. Because the quality of the story is a major influence on transportation, it is often helpful for researchers or health professionals to work with professional writers, animators, or other types of content creators. In our experience, these partnerships work best when the creative side of the team has at least some knowledge about behavioral science principles, and even more importantly, when the creative side is open to feedback and revision from the research side of the team. Writers or other professionals who hold too firmly to their artistic vision at the expense of communicating the educational message are not ideal collaborators; similarly, the more research or health side of the team should also be open to learning from the experience of the experts on the creative side. For example, sometimes researchers may need to adapt or modify their theories to create an intervention that is plausible or resonates with a particular audience, and the creative team may have valuable insights about what will or will not work for creating audience engagement. Clear discussions at the start of the project about expectations for both sides may help avoid problems later. Additionally, keeping in mind the primary purpose of the project may help guide decision-making: if the main goal is theory-testing, the research side of the team should make the final decisions, whereas if the goal is to maintain television ratings or other more entertainment-focused outcomes, the creative team may take priority. If the goal is to create as much attitude or behavior change as possible, there may be more flexibility about trying new creative directions while keeping in mind principles from communication research. A strong partnership can help create the appropriate balance of entertainment and education.

Amount of exposure. It is certainly possible for a single story, even a short one, to change individuals' attitudes and behaviors. This type of change may be especially likely if the story gives the audience new 
information, as opposed to trying to change an existing attitude. However, it is a challenge for one single story to have a large impact on people, especially given the crowded media environment that many people experience today. Therefore, stories that allow for repeated exposure in some way (e.g., multiple episodes of a series; story-based commercials shown more than once; posters that remind people of key episodes or messages from a narrative) may be helpful for longer-term impact.

Importance of pretesting or formative research. Not all stories will work for all audiences. Therefore, it is important to do some preliminary testing of potential entertainment-education storylines or drafts with members of the intended audience or target population. (This may be especially necessary if members of the research or creative team do not come from that population, such as adults writing for children.) This pretesting might involve focus groups or a pilot test with a smaller group of participants. For example, in a recent project examining whether narratives could increase the use of active video games among children, our team tested four potential stories. Just for fun, the members of the team each guessed which story would be the favorite among the children-and we were mostly wrong! Similarly, in the first draft of a narrative game aimed at older adults to promote awareness of heart attack symptoms, we had created a fantasy world complete with magic and wizards. Our focus groups did not care for this approach-they felt that it trivialized the serious issue of heart attacks - and the final version of the game used more realistic, everyday stories instead. The feedback from the target audience at an early stage of the project was essential in shaping the final product and increasing chances of success.

Coherence of story. Although there are many ways to help increase transportation into a story, breaks in the coherence of the story seem to be a primary way of reducing transportation. When a story stops making sense in some way, the audience becomes annoyed or confused. If a character acts in a way that is very different from the way readers expect or if a story takes a completely implausible turn, it is hard for readers to stay immersed in the story. Making sure the plot is coherent is an important way of supporting transportation.

Keep the message central to the plot. As noted above, events that are central to the cause-and-effect structure of the plot are better remembered by audiences. Therefore, the key educational messages may be best delivered by being part of events in the core plot of the story. The story should show the behaviors, consequences, and/or the recommended courses of 
action, rather than relegating them to a less central role in the story. However, creators should be careful to do this in a way that does not evoke reactance from the audience.

\section{REFERENCES}

Appel, M., Gnambs, T., Richter, T., \& Green, M. C. (2015). The transportation scale-short form (TS-SF). Media Psychology, 18(2), 243-266.

Braddock, K., \& Dillard, J. P. (2016). Meta-analytic evidence for the persuasive effect of narratives on beliefs, attitudes, intentions, and behaviors. Communication Monographs, 83(4), 446-467. https://doi.org/10.108 $0 / 03637751.2015 .1128555$

Busselle, R., \& Bilandzic, H. (2009). Measuring narrative engagement. Media Psychology, 12(4), 321-347.

Cacioppo, J. T., Petty, R. E., \& Feng Kao, C. (1984). The efficient assessment of need for cognition. Journal of Personality Assessment, 48(3), 306-307.

Cohen, J. (2001). Defining identification: A theoretical look at the identification of audiences with media characters. Mass communication os society, $4(3), 245-264$.

Dahlstrom, M. F. (2012). The persuasive influence of narrative causality: Psychological mechanism, strength in overcoming resistance, and persistence over time. Media Psychology, 15(3), 303-326. https://doi.org/10.108 $0 / 15213269.2012 .702604$

De Graaf, A., Hoeken, H., Sanders, J., \& Beentjes, J. W. (2012). Identification as a mechanism of narrative persuasion. Communication Research, 39(6), 802-823. https://doi.org/10.1177/0093650211408594

Escalas, J. E. (2004). Imagine yourself in the product: Mental simulation, narrative transportation, and persuasion. Journal of Advertising, 33(2), 37-48.

Green, M. C. (2004). Transportation into narrative worlds: The role of prior knowledge and perceived realism. Discourse Processes, 38(2), 247-266.

Green, M. C., \& Brock, T. C. (2000). The role of transportation in the persuasiveness of public narratives. Journal of Personality and Social Psychology, 79, 701-721.

Green, M. C., \& Brock, T. C. (2002). In the mind's eye: Imagery and transportation into narrative worlds. In M. C. Green, J. J. Strange, \& T. C. Brock (Eds.), Narrative impact: Social and cognitive foundations (pp. 315-341). Mahwah: Lawrence Erlbaum Associates.

Green, M. C., \& Jenkins, K. M. (2014). Interactive Narratives: Processes and Outcomes in User-Directed Stories. Journal of Communication, 64(3), 479-500.

Green, M. C., Kass, S., Carrey, J., Feeney, R., Herzig, B., \& Sabini, J. (2008). Transportation across media: Print versus film comparisons. Media Psychology, $11(4), 512-539$. 
Kreuter, M. W., Green, M. C., Cappella, J. N., Slater, M. D., Wise, M. E., Storey, D., et al. (2007). Narrative communication in cancer prevention and control: A framework to guide research and application. Annals of Behavioral Medicine, $33(3), 221-235$.

Leung, M. M., Green, M. C., Tate, D. F., Cai, J., \& Ammerman, A. (2017). Fight for Your Right to Fruit (C): Psychosocial outcomes of a manga comic promoting fruit consumption in middle-school youth. Health Communication, $32(5), 533-540$.

Lu, A. S., Green, M. C., \& Thompson, D. (2019). How to increase children's physical activity through narrative game design: An exploratory thematic analysis. Journal of Medical Internet Research: Serious Games, 7(4), el6031. https:// doi.org/10.2196/16031

Mar, R. A., \& Oatley, K. (2008). The function of fiction is the abstraction and simulation of social experience. Perspectives on psychological science, 3(3), 173-192.

Mazzocco, P. M., Green, M. C., Sasota, J. A., \& Jones, N. W. (2010). This story is not for everyone: Transportability and narrative persuasion. Social Psychology and Personality Science, 1(4), 361-368.

Murphy, S. T., Frank, L. B., Chatterjee, J. S., \& Baezconde-Garbanati, L. (2013). Narrative versus nonnarrative: The role of identification, transportation, and emotion in reducing health disparities. Journal of Communication, 63(1), 116-137.

Nabi, R. L., \& Green, M. C. (2015). The role of a narrative's emotional flow in promoting persuasive outcomes. Media Psychology, 18(2), 137-162.

Ophir, Y., Sangalang, A., \& Cappella, J. N. (2021). The emotional flow hypothesis in entertainment-education narratives: Theory, empirical evidence, and open questions. In L. B. Frank \& P. Falzone (Eds.), Entertainment-education behind the scenes: Case studies for theory and practice. Hampshire: Palgrave Macmillan.

Simons, J. J. P., \& Green, M. C. (2013). Distracted by details: Narrative influence following conflicting stories. Media Psychology, 16(2), 221-243.

Strange, J. J., \& Leung, C. C. (1999). How anecdotal accounts in news and in fiction can influence judgments of a social problem's urgency, causes, and cures. Personality and Social Psychology Bulletin, 25(4), 436-449.

Tukachinsky, R. (2014). Experimental manipulation of psychological involvement with media. Communication Methods and Measures, 8(1), 1-33.

Van Laer, T., de Ruyter, K., Visconti, L. M., \& Wetzels, M. (2014). The extended transportation-imagery model: A meta-analysis of the antecedents and consequences of consumers' narrative transportation. Journal of Consumer Research, $40(5), 797-817$.

Vaughn, L. A., Childs, K. E., Maschinski, C., Paul Niño, N., \& Ellsworth, R. (2010). Regulatory fit, processing fluency, and narrative persuasion. Social and Personality Psychology Compass, 4(12), 1181-1192. 
Vezzali, L., Stathi, S., Giovannini, D., Capozza, D., \& Trifiletti, E. (2015). The greatest magic of Harry Potter: Reducing prejudice. Journal of Applied Social Psychology, 45(2), 105-121.

Walter, N., Murphy, S. T., Frank, L. B., \& Baezconde-Garbanati, L. (2017). Each medium tells a different story: the effect of message channel on narrative persuasion. Communication Research Reports, 34(2), 161-170.

Open Access This chapter is licensed under the terms of the Creative Commons Attribution-NonCommercial-NoDerivatives 4.0 International License (http:// creativecommons.org/licenses/by-nc-nd/4.0/), which permits any noncommercial use, sharing, distribution and reproduction in any medium or format, as long as you give appropriate credit to the original author(s) and the source, provide a link to the Creative Commons licence and indicate if you modified the licensed material. You do not have permission under this license to share adapted material derived from this chapter or parts of it.

The images or other third party material in this chapter are included in the chapter's Creative Commons licence, unless indicated otherwise in a credit line to the material. If material is not included in the chapter's Creative Commons licence and your intended use is not permitted by statutory regulation or exceeds the permitted use, you will need to obtain permission directly from the copyright holder. 